\title{
EQUATORIAL SOLITARY WAVES \\ Part 4. Kelvin solitons in a shear flow
}

\author{
JOHN P. BOYD \\ Department of Atmospheric and Oceanic Science, University of Michigan, \\ 2455 Hayward Avenue, Ann Arbor, MI 48109 (U.S.A.)
}

(Received October 7, 1983; revised February 29, 1984; accepted March 26, 1984)

\begin{abstract}
Boyd, J.P., 1984. Equatorial solitary waves. Part 4. Kelvin solitons in a shear flow. Dyn. Atmos. Oceans, 8: 173-184.

It is shown that a mean flow with shear makes the Kelvin wave dispersive. This in turn modifies its nonlinear behavior and makes it necessary to replace the one-dimensional advection equation derived in an earlier work of the author's by the Korteweg-deVries equation instead. The frontogenesis predicted in the earlier paper will still occur, but the wave breaking will not. Instead, once a steep front has formed, it will disintegrate into a train of solitary waves. These then propagate towards the east at a faster-than-linear rate. It is also shown that Kelvin solitary waves will have much smaller zonal widths than Rossby solitons of the same height; "round" Kelvin solitary waves (equal zonal and latitudinal width) are to be expected, and are fully consistent with the small amplitude, weak dispersion theory. An interesting implication of the Korteweg-deVries model is that the peak signal from a nonlinear Kelvin wave packet may be roughly double that of a linear Kelvin wavetrain.
\end{abstract}

\section{INTRODUCTION}

This paper is the seventh by the author in a series dealing with nonlinear equatorial waves (Boyd 1980a,b; 1983a,b,c; 1984) and the fourth of the series to describe equatorial solitary waves. The previous six articles assumed that the mean state was one of rest, thus ignoring mean currents. Though obviously unrealistic, this was justified both by the complexity of the theory even without mean flow and by the fact that weak shear will not qualitatively alter most results. To be sure, a weak mean current will change the latitudinal structure a little, and add small corrections to the speed and amplitude, but it will not preclude or prevent solitary waves unless the shear is so strong as to create critical latitudes or instability - and perhaps not 
even then. Johnson (1972) gives a good discussion of solitary water waves in weak shear flows; Redekopp (1977) describes Rossby solitons with critical latitudes and (weak) instability.

The Kelvin wave, however, is a special case. In the absence of shear, it is nondispersive and therefore cannot form solitary waves at all, as discussed by Boyd (1980a). By creating dispersion, weak shear drastically modifies even the qualitative behavior of the Kelvin wave. Instead of forming steep fronts and eventually breaking, a Kelvin wave in shear will evolve into solitary waves of Korteweg-deVries type instead.

In the previous articles in this series, the singular perturbation theory which describes each different type of nonlinear equatorial wave has been worked out in careful detail. Here, the derivation will be brief, and the formal treatment of the problem will be outlined more in words than with equations. The reason is that the nonlinear term in the Korteweg--deVries equation has already been calculated (Boyd 1980a). The other half of our task, which is to evaluate the dispersive term of the Korteweg-deVries equation, can be done in a strictly linear calculation: a perturbative treatment of shear effects on a Kelvin wave. Complete results are given in the appendix, but we actually only need the eigenvalue $c$, which can be determined via the Rayleigh-Schrödinger formula for general current profiles without calculating the eigenfunction at all.

The next section derives the Korteweg-deVries equation for the Kelvinwave-in-shear. Section 3 discusses the connection between the KortewegdeVries solutions and those of the one-dimensional advection equation which describes Kelvin waves in the absence of shear. The final section is a summary and prospectus.

\section{DERIVATION OF THE KORTEWEG-DEVRIES EQUATION}

The formal perturbation theory begins with the following assumptions:

(i) The wave amplitude is $O(\epsilon)$ where $\epsilon \ll 1$,

(ii) the mean current $U(y)$ is $O(S)$ where $S \ll 1$, and

(iii) $S^{2} / L^{2} \sim O(\epsilon)$,

where $L$ is the zonal length scale. The first two assumptions allow both the nonlinearity and the dispersion-inducing shear flow to be handled through perturbation theory. The third assumption is necessary so the effects of nonlinearity and dispersion can balance to create a solitary wave. When the shear is much stronger than allowed by (iii), the Kelvin wave will simply disperse; when the shear is very weak in comparison to the nonlinearity, the wave will steepen and develop a front (Boyd, 1980a).

One striking difference from the Rossby solitary wave is that we have not assumed that the zonal length scale $L$ is large in comparison to one. For 
Rossby waves, this is essential because with or without mean current shear, Rossby waves are weakly dispersive only when the zonal scale is large in comparison to one. When $L$ is $O(1)$, Rossby waves-except for the nonlinear wave packets discussed in Boyd (1983a) - disperse too rapidly for the dispersion to be balanced by a weak nonlinearity. None of these three order-of-magnitude assumptions affects the actual numerical calculations, but each is essential for the logical consistency of the final result.

The zeroth order perturbation equations are simply those for a linear Kelvin wave with no mean current with the familiar solution

$u^{0}=A(x, t) e^{-(1 / 2) y^{2}}$

$\varphi^{0} \equiv u^{0}$

$v^{0} \equiv 0$

where all quantities have been nondimensionalized as in Boyd (1980a) and where $A(x, t)$ satisfies the amplitude equation

$A_{t}+c A_{x}=0+O\left(\epsilon, S^{2}\right)$

where the phase speed $c=1$ to lowest order. At $O(S) \sim O\left(\epsilon^{1 / 2}\right)$, the nonlinear terms do not appear, and one is merely calculating the first-order shear-induced corrections to the zeroth-order wave. At second order, $O(\epsilon)$ $\sim O\left(S^{2}\right)$, the nonlinear terms and the second order shear-induced corrections both appear. The nonsecularity condition upon the second order forcing then gives the Korteweg-deVries (KdV) equation in the form

$A_{t}+c_{0} A_{x}+D A A_{x}+F(S) A_{x x x}=0$

where $D$ is a numerical constant and $F$ is a function only of the shape and form of the mean current. Note that (2.5) differs slightly from the form of the Korteweg-deVries equation given in Boyd (1980a) in that it is written directly in terms of $x$ and $t$ rather than in coordinates moving at the linear, nondispersive phase speed $c_{0}$.

The key observation is that the nonlinearity and dispersion enter (2.5) in an independent, additive fashion. (They become coupled only when the perturbation theory is carried to higher order.) In particular, the $O(S)$ shear corrections to the zeroth order Kelvin wave do not appear in the nonlinear terms at $O(\epsilon)$; such corrections enter only at $O(\epsilon S)$. Thus, the nonlinear contribution to (2.5) must be exactly the same as in Boyd (1980a), which neglected mean shear. The earlier work gives

$D=(3 / 2)^{1 / 2}=1.2247$

To put it another way, the nonlinear coefficient in (2.5) must be the same as in the nondispersive Kelvin treatment of Boyd (1980a) so that the KdV 
equation will reduce to the one-dimensional advection equation of the earlier paper in the limit $S \rightarrow 0$. Similarly, the Korteweg-deVries equation must correctly reproduce the linear dispersion relation for a simple plane wave in the limit of infinitesimal amplitude.

Although Boyd (1980b) gave only numerical coefficients, this need for consistency with the linear dispersion relation for a sine wave gives an analytic formula for the third derivative term of the $\mathrm{KdV}$ equation for Rossby waves, too, and it is illuminating to derive this first before proceeding to the slightly harder case of the Kelvin in shear. For either wave type, the coefficient of the $\mathrm{KdV}$ third derivative can be obtained merely by calculating the phase speed $c$ as a power series in the dispersion parameter, which, for Rossby waves without shear, is the wavenumber $k$. By substituting a power series in $k$ into the usual cubic dispersion relation for equatorial waves, one finds

$c \simeq \frac{-1}{(2 n+1)}+k^{2}\left\{\frac{1}{(2 n+1)^{2}}\left[1-\frac{1}{(2 n+1)^{2}}\right]\right\}+O\left(k^{4}\right)$

where $n$ is the meridional mode number of the Rossby wave. Since the Korteweg-deVries equation must be consistent to $O(\epsilon)$ for all ultra-long Rossby waves of zonal scale $L=O\left(\epsilon^{1 / 2}\right)$, whether they are sine waves or solitons, it follows that the coefficient of the third derivative of the equation must equal the negative of the second order term in the expansion of $c$ in the dispersion parameter, i.e.

$F=-\left[1-1 /(2 n+1)^{2}\right] /(2 n+1)^{2}$

which agrees with the numbers in (3.35e), (3.36e), and (3.37e) of Boyd (1980b) for $n=1,3$, and 5 to all decimal places given.

A similar expansion generates the third derivative coefficient $F$ for the Kelvin wave, but the dispersion parameter is now the shear strength $S$ rather than the wavenumber. The perturbative calculation of the effects of shear on the phase speed $c$ is very similar to that given in Boyd $(1978 a, b)$, so the details will not be given here. For the general parabolic wind profile

$U(y)=U_{0}+S\left[\Gamma y+\Delta y^{2}\right]$

which is geostrophically balanced by the mean height field

$H(y)=1-S\left[\Gamma y^{3} / 3+\Delta y^{4} / 4\right]$

one finds

$$
\begin{aligned}
c= & \left\{1+U_{0}+S \Delta(13 / 32)+S^{2}\left[\Gamma^{2}(175 / 576)+\Delta^{2}(473 / 2048)\right]\right\} \\
& -k^{2} S^{2}\left\{\Gamma^{2}(83 / 288)+\Delta^{2}(53 / 512)\right\}
\end{aligned}
$$


The terms in the first set of braces in (2.11) are corrections to the linear, nondispersive phase speed $c_{0}$ that appears in the Korteweg-deVries equation. The terms in the second set of braces are the dispersion induced by the shear. The Korteweg-deVries equation is consistent with (2.11) if and only if it takes the form

$A_{t}+c_{0} A_{x}+1.225 A A_{x}+F A_{x x x}=0$

where

$c_{0}=1+U_{0}+0.406 \Delta+0.304 \Gamma^{2}+0.231 \Delta^{2}$

$F=0.288 \Gamma^{2}+0.104 \Delta^{2}$

Note that since $S$ is a dummy parameter introduced merely to order the magnitudes of different terms, we are now free to dispose of it by setting $S=1$ in (2.12). The validity of the perturbation theory then requires that the nondimensional parameters $\Gamma$ and $\Delta$ both be small in comparison to unity, however.

The Korteweg-deVries equation (2.12) is the principal result of the paper, but there are some technical comments that should be made before discussing the solutions $A(x, t)$ of $(2.12)$ in the next section.

First, because the parabolic flow is unbounded, the mean depth $H(y)$ will vanish at some latitude $y$ (unless $\Gamma=0$ and $\Delta<0$ ) and the mean current $U(y)$ will create a critical latitude where it equals the phase speed. Either circumstance would seem to make nonsense of our perturbation scheme. As stressed in Boyd (1978a,b), however, the breakdown of the assumption of the "smallness" of the perturbation for linear and parabolic currents (or any unbounded flow) for large $y$ merely causes the perturbation series to be asymptotic rather than convergent because for small $\Gamma$ and $\Delta$, this breakdown occurs only where the amplitude of the wave is exponentially small anyway. An alternative way of conceptualizing this is to imagine that the parabolic profile (2.9) is really a near-equatorial approximation to a bounded flow in which $U(y)$ is proportional to a Gaussian or a hyperbolic tangent function or something similar. We make only an exponentially small error in using the parabola (2.9) to compute $c$ (and therefore $F$ ) if the approximation of the parabola to the bounded flow breaks down only for large $|y|$.

Second, for more general currents, even those varying too rapidly near the equator to be approximated by the parabola, we can infer the form of the result without performing any actual calculations. Omitting the details, it can be shown, by dividing the equations by $k$ and introducing $\bar{v} \equiv v / k$, that for any current $U(y)$, there is perturbative dispersion relation of the form

$c=c_{0}(S$ only $)-F k^{2} S^{2}+O\left(S^{3}\right)$ 
where $F$ is a numerical constant. Since this is identical in form with (2.11), we have verified that we will always obtain the Korteweg-deVries equation, regardless of the shape of $U(y)$.

The third and final comment is to note that although the perturbed $u, v$, and $\varphi$ are given in the appendix for completeness, it is quite unnecessary to compute them to obtain the dispersive coefficient of the $\mathrm{KdV}$ equation. Since the unperturbed equations for equatorial waves are a self-adjoint system, one can calculate the eigenvalue directly by using the familiar Rayleigh-Schrödinger perturbation formula, which can be found in any quantum mechanics text. A readable reference is Merzbacher (1970). The only complications (minor ones) are that the shear terms, which play the role of the perturbation potential energy $\mathrm{V}$, are a non-self-adjoint operator, and that the unperturbed operator is a $3 \times 3$ matrix operator rather than a single equation. The mechanics of the corresponding vector eigenfunctions and inner products are discussed in the appendix of Cane and Sarachik (1979) and in Boyd (1983a). The point is that it is straightforward to obtain the coefficient of the third derivative of the Korteweg-deVries equation for quite arbitrary currents; the parabola was used only for convenience.

\section{KELVIN SOLITARY WAVES}

The general solution of the Korteweg-deVries equation has been discussed in Boyd (1980b), so here we will concentrate only upon solitary waves. The most important qualitative conclusion is that since the nonlinear and dispersive coefficients of the $\mathrm{KdV}$ equation for Kelvin waves in shear are of the same sign, as true also of the Rossby equations of Boyd (1980b), the solitary waves will be of elevation only. Put another way, Kelvin solitons of the first baroclinic mode will increase the depth of the thermocline. Strictly speaking, we can assert this with surety only for the parabolic flow (2.9), but since inclusion of both symmetric and antisymmetric mean flow components still leads to a dispersive coefficient of the $\mathrm{KdV}$ equation which is positive definite, Kelvin solitons of elevation are to be expected even for mean flows considerably distorted from the parabola.

The difference in sign from Rossby waves - both coefficients are positive for the Kelvin wave, but negative for Rossby waves-implies that the qualitative effects of dispersion and nonlinearity will be the same in either case, but with the direction of propagation reversed. The Rossby solitons travel to the west at a greater-than-linear rate, and the dispersive wavetrain, if any, is left trailing behind. In a similar way, the Kelvin soliton travels to the east at a greater-than-linear speed, and if the initial condition excites a wavetrain as well as a solitary wave, the wavetrain will lag behind to the west of the soliton. 
The lowest order solution is

$u=A(x, t) e^{-(1 / 2) y^{2}}$

$\varphi \equiv u$

$v \equiv 0$

where $A(x, t)$ satisfies the $\mathrm{KdV}$ equation (2.12). The single soliton solutions are

$A=9.80 F B^{2} \operatorname{sech}^{2}\left[B\left(x-\left\{c_{0}+4 F B^{2}\right\} t\right)\right]$

where $F$ is the coefficient of the third derivative term in the Korteweg-deVries equation and $c_{0}$ is the phase speed of a Kelvin wave in the limit of infinitesimal amplitude and infinite zonal scale. For a fixed mean current, the solitons are a one-parameter family with the pseudowavenumber $B$ as the parameter. Figure 1 shows the shape of the velocity and height fields for a

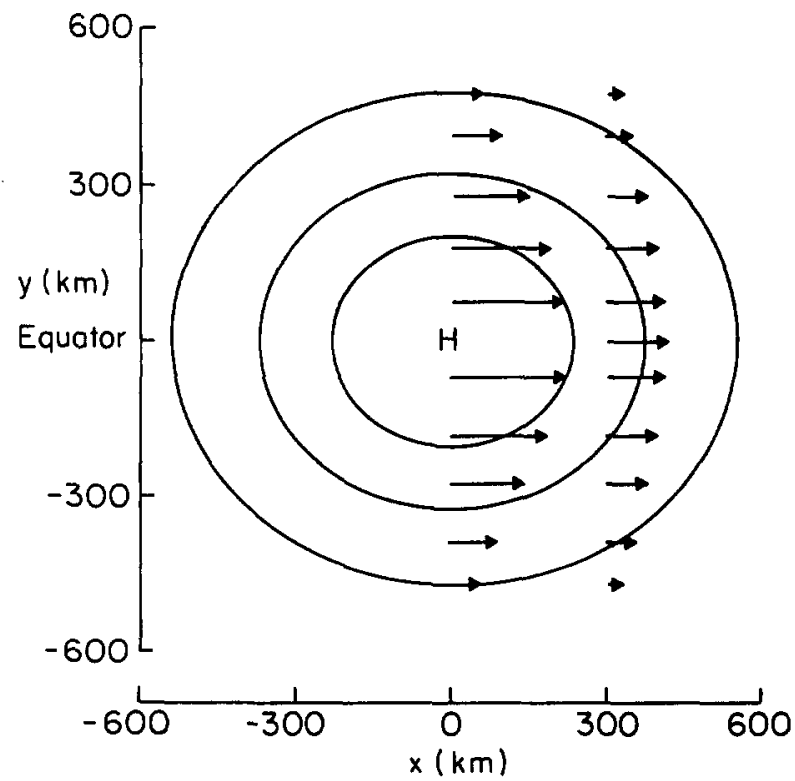

Fig. 1. The height and horizontal wind field for a typical pseudowavenumber of $B=0.75$. The nondimensional horizontal length scale has been taken as $300 \mathrm{~km}$ (first baroclinic mode). The contours of constant height are $0.75,0.5$, and 0.25 of the peak marked by the " $H$ ". The magnitude and direction of the soliton velocities are marked by arrows. Both the amplitude and velocity scales have been left arbitrary since the magnitude of $u$ and $\varphi$ depend on the strength and form of the mean current. The shape of the $u$ and $\varphi$ fields, however, depends only upon the zonal width, and thus can be graphed unambiguously. The Kelvin soliton propagates towards the east at a superlinear rate. 
particular choice of $B$. Equations (3.1)-(3.4) show that the shape is determined solely by $B$; the amplitude and nonlinear phase speed correction are what are sensitive to $F$, and therefore to the mean flow $U(y)$.

In view of the intrinsic variability of the currents and the limitations of approximating the currents by a parabola and the actual stratified ocean by a one-layer model, it is foolish to discuss the Kelvin solitary waves except in a qualitative, order-of-magnitude sense. Although it will therefore be necessary to choose parameter values somewhat arbitrarily, one can nonetheless obtain a feeling for the possibilities.

The magnitude of the shear is constrained by the need for (i) barotropic stability, which requires $\Delta \leqslant 0.5$ and (ii) no critical latitudes too close to the equator, which implies we should (roughly) restrict $\Gamma \leqslant 0.5$ also. (The Kelvin wave instability of Boyd and Christidis, 1982, 1983, is negligible for such weak shears.) For these limiting values, one finds

$$
\begin{aligned}
& \Delta=0.5, \Gamma=0: A(x, t)=0.255 B^{2} \operatorname{sech}^{2}\left[B\left(x-\left\{1.26+0.104 B^{2}\right\} t\right)\right] \\
& \Gamma=0.5, \Delta=0: A(x, t)=0.706 B^{2} \operatorname{sech}^{2}\left[B\left(x-\left\{1.08+0.288 B^{2}\right\} t\right)\right]
\end{aligned}
$$

These limiting shears are not particularly small in comparison to one, of course, as required for the validity of the perturbation theory, but Boyd (1984) has shown that the Korteweg-deVries theory for the $n=1$ Rossby soliton is accurate even when the solitary wave is so large that it has a closed region of entrained, recirculating fluid and behaves like a modon. This is quite common in singular perturbation theory (Bender and Orszag, 1978, give other examples), so it is reasonable to discuss Kelvin solitons for the shears of eq. 3.5.

For $B=1$, for example, which for the first baroclinic mode corresponds to a zonal half-width of about $500 \mathrm{~km}$ where the half-width is the distance between the two points where $A=1 / 2$ of its maximum value, one finds that the phase speed corrections are modest. The solitons, however, are of rather large amplitude with the ridge in linear shear almost doubling the depth of the thermocline.

In weaker shear, solitons of this same width are much smaller, decreasing as the square of the shear strength for fixed zonal scale. Conversely, if the shear is fixed and the pseudowavenumber is reduced, the soliton amplitude again decreases as the square of $B$. One is left with the conclusion that unless either $B$ is $O(1)$ and the shears are $O(1 / 2)$ or alternatively, $B \gg 1$ for weaker shears, the solitons will be quite small. As stressed earlier, derivations of the Korteweg-deVries equation in other contexts usually require that the zonal scale be a "slow" length scale so that the solitons are elongated in the zonal direction. Boyd (1983d) shows that this perturbative requirement need not be taken too seriously for the $n=1$ Rossby wave, which can be round and 
modon-like and yet still be accurately described by the lowest order theory of Boyd (1980b). For Kelvin solitons, this requirement does not exist even in principle. Since $B=0.75$ (illustrated in Fig. 1) makes the zonal and latitudinal half-widths identical, it is clear from (3.5) that Kelvin solitons must be round, or perhaps even wider in latitude than longitude, to have large amplitude.

Nonetheless, even in weak shear, the current-induced dispersion will still profoundly influence wave behavior by preventing the frontogenesis and wave-breaking which, as discussed in Boyd (1980a), would otherwise inevitably occur along the crest of the Kelvin wave. Whitham (1974) gives a good discussion of what happens when the Korteweg-deVries equation with weak dispersion, i.e., a small value of the third derivative coefficient $F$, is given a large initial condition. During the early stages of the evolution of the flow, the third derivative is negligible and the crest of the wave does develop a steep front. Even if the initial scale of this disturbance is very large, however, this very frontogenesis creates the small zonal scales which are necessary for dispersion to be important. Instead of rolling over and becoming multi-valued, the wave does not break, but rather the front breaks up into an assortment of narrow (and narrowly spaced) solitons as shown schematically in Fig. 2.

This qualitative behavior is remarkably indifferent to the actual size of the dispersion coefficient. Decreasing $F$ still further causes the disintegration of the front into solitons, which occurs very rapidly just before the nondispersive front would break, to happen later and still more rapidly, and the front is chopped up into a larger number of narrower solitary waves. Soliton formation must occur, however, and no later than the time of breaking $t_{\mathrm{B}}$ calculated by setting $F=0$ and solving the resulting nondispersive equation. Thus, even a weak mean current would turn Kelvin wave-breaking into Kelvin solitons.

Whitham (1974) gives a full description of this process, but it is useful to quote a couple of quantitative facts. First, the height of the tallest soliton is bounded by twice the maximum positive value of $A(x, t=0)$. Second, the number of solitons generated from an initial condition is roughly given by

$N=\frac{0.144}{F^{1 / 2}} \int_{-\infty}^{\infty}|A(x, t=0)|^{1 / 2} \mathrm{~d} x$

provided that the predicted number is large. Equation 3.6 shows explicitly just what was claimed above: as the dispersion coefficient $F$ is reduced, more solitons are generated from the same, fixed initial condition, which is obviously possible only if the solitons have narrower zonal scales. In the limit $F \rightarrow 0$, of course, viscous effects will certainly become important, but an attempt to estimate precisely on what scale would take us too far afield. 


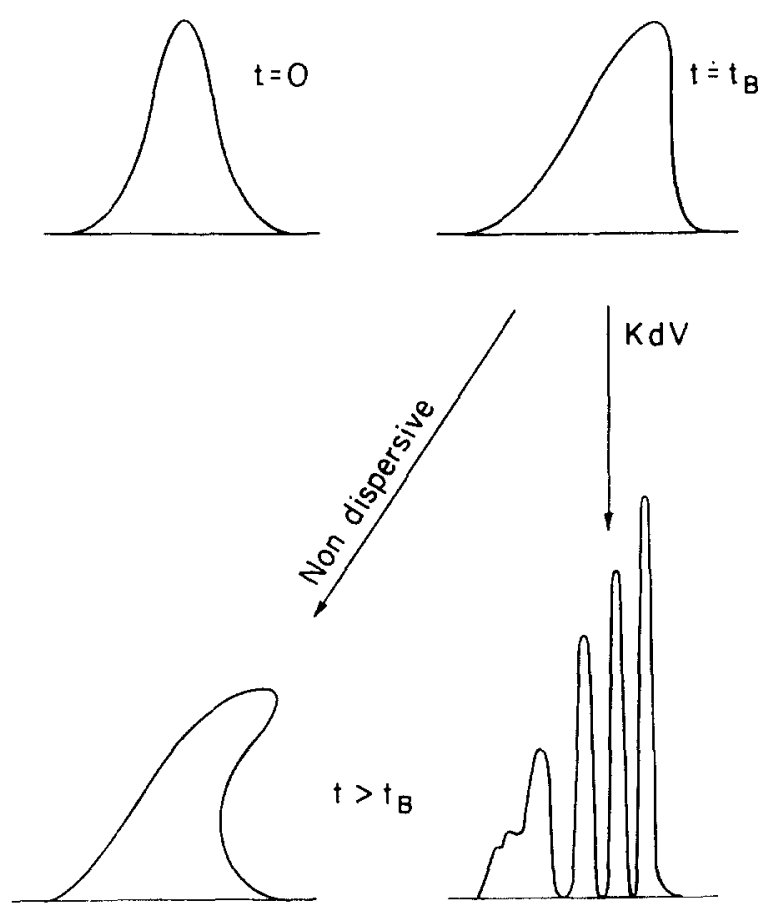

Fig. 2. Schematic solution of the Korteweg-deVries equation for very weak dispersion. During the early stages, the leading edge of the wave packet steepens while the trailing edge flattens, just as in the nondispersive case illustrated by Fig. 1 of Boyd (1980a). This continues until $t$ approximately equals $t_{\mathrm{B}}$, which is the time of wave-breaking obtained by ignoring the dispersion completely and solving the one-dimensional advection instead equation. After this, however, the wave behavior is wildly different: the two alternatives for $t>t_{\mathrm{B}}$ are shown in the lower two panels. If the wave is truly nondispersive, then the solution becomes multi-valued, i.e., the wave breaks. If dispersion is present, even weakly, the wave packet will break up into a number of tall, narrow solitons. The height of the largest solitary wave will be roughly double the maximum wave amplitude at $t=0$ even though there is no increase of the wave amplitude whatsoever during the nondispersive evolution of the wave, either before or after breaking.

\section{SUMMARY AND CONCLUSIONS}

It has been shown that the shear of a mean current, even weak shear, can profoundly alter the behavior of a Kelvin wave by replacing breaking with the formation of solitary waves instead. Unlike Rossby solitary waves, which are usually much wider in $x$ than in latitude, Kelvin solitons, except those of very small amplitude, must have rather small zonal scales with zonal halfwidths of the order $500-1000 \mathrm{~km}$ or smaller.

The nonlinear coefficient of the Korteweg-deVries equation for Kelvin waves is identical with that of the one-dimensional advection equation of Boyd (1980a); it is not affected by the mean current to this order. The 
dispersive coefficient of the $\mathrm{KdV}$ equation is explicitly calculated in closed form for a general parabolic current. It can be computed for more general currents by borrowing the familiar Rayleigh-Schrödinger perturbation formula from quantum mechanics to calculate the phase speed $c$ for an ordinary, linear sine wave to second order in the strength of the shear; the dispersive coefficient is independent of the nonlinearity to this order.

The shear of the mean flow will affect the Rossby solitons of Boyd (1980b) also, but because long Rossby waves are already dispersive, even for a resting mean state, the only effect will be to alter the numerical values a little without changing the qualitative behavior of the waves. The mean flow has a much more profound effect on Kelvin waves because Kelvin waves have no dispersion when a latitudinally varying mean current is absent.

\section{ACKNOWLEDGMENTS}

This work was supported by the National Science Foundation under grants OCE8108530 and OCE8305648.

I am grateful to Dr. Hal Marshall for suggesting this problem and for helpful discussions during its completion. I also thank the two anonymous reviewers for useful suggestions.

APPENDIX: THE FULL PERTURBATIVE SOLUTION

The parabolic wind profile

$$
U(y)=\Gamma y+\Delta y^{2}
$$

is geostrophically balanced by the height

$$
H(y)=1-\frac{\Gamma y^{3}}{3}-\frac{\Delta y^{4}}{4}
$$

The nondimensional dispersion relation to second order is

$$
\begin{aligned}
c= & 1+(13 / 32) \Delta+\Gamma^{2}\left\{175 / 576-k^{2}(83 / 288)\right\} \\
& +\Delta^{2}\left\{473 / 2048-k^{2}(53 / 512)\right\}
\end{aligned}
$$

The zeroth order fields are

$$
\begin{aligned}
& u^{0}=\phi^{0}=e^{-(1 / 2) y^{2}} \\
& v^{0} \equiv 0
\end{aligned}
$$


The first order corrections are

$$
\begin{aligned}
u^{1}= & \Gamma y\left\{\left(1-5 k^{2}\right) / 6+\left(2+k^{2}\right) y^{2} / 18-y^{4} / 30\right\}+\Delta\left\{\left(46 k^{2}-83\right) / 256\right. \\
& \left.+\left(25-26 k^{2}\right) y^{2} / 64+\left(5+2 k^{2}\right) y^{4} / 64-y^{6} / 48\right\} \\
\phi^{1}= & \Gamma y\left\{-5 k^{2} / 6+\left(k^{2}-1\right) y^{2} / 18-y^{4} / 30\right\} \\
& +\Delta\left\{\left(21+46 k^{2}\right) / 256+\left(13-26 k^{2}\right) y^{2} / 64\right. \\
& \left.+\left(2 k^{2}-3\right) y^{4} / 64-y^{6} / 48\right\} \\
v^{1}= & \Gamma\left(5-y^{2}\right) / 6+\Delta y\left(13-2 y^{2}\right) / 16
\end{aligned}
$$

\section{REFERENCES}

Bender, C.M. and Orszag, S.A., 1978. Advanced Mathematical Methods for Scientists and Engineers. McGraw-Hill, New York, pp. 514-516 and 554-557.

Boyd, J.P., 1980a. The nonlinear equatorial Kelvin wave. J. Phys. Oceanogr., 10: 1-11.

Boyd, J.P., 1980b. Equatorial solitary waves, Part 1. Rossby solitons. J. Phys. Oceanogr., 10: $1699-1717$.

Boyd, J.P., 1983a. Equatorial solitary waves, Part 2. Envelope solitons. J. Phys. Oceanogr., 13: $428-449$.

Boyd, J.P., 1983b. Long wave/short wave resonance in equatorial waves. J. Phys. Oceanogr., 13: $450-458$.

Boyd, J.P., 1983c. Second harmonic resonance in equatorial waves. J. Phys. Oceanogr., 13: $459-466$.

Boyd, J.P., 1984. Equatorial solitary waves, Part 3. Modons. J. Phys. Oceanogr., 13: in press.

Boyd, J.P. and Christidis, Z.D., 1982. Low wavenumber instability on the equatorial betaplane. Geophys. Res. Lett., 9: 769-772.

Boyd, J.P. and Christidis, Z.D., 1983. Instability on the equatorial beta-plane. In: J.C.J. Nihoul (Editor), Hydrodynamics of the Equatorial Ocean. Elsevier, New York, pp. $335-351$.

Cane, M.A. and Sarachik, E.S., 1979. Forced baroclinic ocean motions, III. The linear equatorial basin case. J. Mar. Res., 37: 355-358.

Johnson, R.S., 1972. Some numerical solutions of a variable-coefficient Korteweg-deVries equation (with applications to solitary wave development on a shelf). J. Fluid Mech., 54: $81-91$.

Merzbacher, E., 1970. Quantum Mechanics, 2nd. edn. John Wiley, New York, 615 pp. (see pp. 413-420).

Redekopp, L.G., 1977. On the theory of solitary Rossby waves. J. Fluid Mech., 82: 725-745.

Whitham, G.B., 1974. Linear and Nonlinear Waves. John Wiley, New York, 636 pp. (see pp. 598-599). 